\title{
Forscher streiten über die Bedeutung von Innovationen
}

Wie wichtig sind Neuerungen und Novitäten für Unternehmen wirklich? Darüber streiten zwei Professoren der Harvard University in aller Öffentlichkeit, wie die FAZ am 7.07.2014 in ihrem mit „Sie schimpfen wie die Rohrspatzen“ überschriebenen Beitrag schildert. Im Streit liegen die preisgekrönte Geschichtsprofessorin Jill Lepore und der Management-Guru Clayton Christensen. Letzterer hat in seinem Werk „The Innovator's Dilemma“ von 1997 seine Theorie der „disruptiven Technologie“ erörtert. Seine Ausführungen, wie Unternehmen mit neuen Techniken alte Zustände und Branchenführer aushebeln können, sind für viele Unternehmen noch heute Gesetz.

$\mathrm{Zu}$ Unrecht, findet Lepore. In einem Essay für das Magazin „The New Yorker“ nimmt sie die Theorien von Christensen Schritt für Schritt auseinanderschreibt. Im Ergebnis prangert sie den Hype um Innovationen an, schreibt von Hysterie und Überbewertung. Christensen schäumt vor Wut und erläutert in einem Interview mit Bloomberg-Businessweek noch einmal die Grundpfeiler seiner Lehre und warum auch innova- tive Unternehmen anhand der wachsenden Konkurrenz mit preiswerten Erzeugnissen am Ende scheitern können.

Diese Auseinandersetzung einfach als Streit zwischen zwei Wissenschaftlern abzutun, wäre zu einfach angesichts der Summe, die Unternehmen jährlich für Forschung und Entwicklung aufwenden. Die FAZ verweist auf Erhebungen des amerikanischen Forschungsinstituts Batelle und des R\&DMagazines, nach denen im letzten Jahr weltweit 1,6 Billionen Dollar hierfür ausgegeben wurden - so viel wie noch nie. Unternehmen werden daher den Streit weiter mit Interesse verfolgen.

Mehr zum Thema:

Noé, M. (2013): Innovation 2.0 - Unternehmenserfolg durch intelligentes und effizientes Innovieren, Wiesbaden,

SIsp www.springerprofessional.de/ 4704436

Vera Treitschke, Wiesbaden

\section{Alleskönner Biltroller zunehmend gefragt}

Unternehmen wünschen sich verstärkt Mitarbeiter, die sowohl im Finanz- als auch im Rechnungswesen zuhause sind. Dies ist eine der zentralen Erkenntnisse aus der aktuellen Gehaltsanalyse des Bundesverbandes der Bilanzbuchhalter und Controller e.V. (BVBC) und des Personaldienstleisters Hays AG, an der rund 2.900 Fach- und Führungskräfte teilgenommen haben.

Gute Berufschancen haben Biltroller vor allem in mittelständischen Unternehmen. Diese Unternehmen sehen es als Vorteil an, wenn sie nur eine Person haben, mit der sie alle betriebswirtschaftlichen Dinge besprechen und die diese gegenüber Dritten wie z. B. Banken vertreten kann. Zudem müssen sie nur eine Fachkraft bezahlen. Aber auch Großunternehmen erwarten von ihren Controllern zunehmend, dass diese bereits im Rechnungswesen an Abschlüssen mitgearbeitet haben und Bilanzen auch interpretieren können. Heute sind Unternehmen mehr denn je darauf angewiesen, dass das Controlling und das Finanz- und Rechnungswesen Hand in Hand arbeiten. Bilanzbuchhalter und Controller sollten also möglichst Erfahrungen in dem jeweils anderen Bereich sammeln und diese durch entsprechende Qualifikationen belegen.

Die Ergebnisse der Gehaltsanalyse wurden auf der Kongressmesse ReWeCo in Bad Soden erstmals der Öffentlichkeit vorgestellt und stießen dort bei den Fachbesuchern auf reges Interesse.

Mehr zum Thema:

Rieg, R./Gruber, T./Reißig-Thust, S. (2013): Der Biltroller im Mittelstand - Controlling und Bilanzierung in einer Hand, in: Controlling \& Management Review, 57 (SH 2), S. 62-69,

SSTw www.springerprofessional.de/4691252 